\title{
Inhibition of ICAM2 induces radiosensitisation in oral squamous cell carcinoma cells
}

\author{
T Ishigami', K Uzawa",', K Fushimi', K Saito', Y Kato', D Nakashima', M Higo', Y Kouzu', H Bukawa², \\ T Kawata ${ }^{3}, \mathrm{H}$ Ito $^{3}$ and $\mathrm{H}$ Tanzawa ${ }^{1,2}$ \\ 'Department of Clinical Molecular Biology, Graduate School of Medicine, Chiba University, I-8-I Inohana, Chuo-ku, Chiba 260-8670, Japan; ${ }^{2}$ Division of \\ Dentistry and Oral-Maxillofacial Surgery, Chiba University Hospital, 1-8-I Inohana, Chuo-ku, Chiba 260-8670, Japan; ${ }^{3}$ Department of Radiology, \\ Graduate School of Medicine, Chiba University, I-8-I Inohana, Chuo-ku, Chiba 260-8670, Japan
}

\begin{abstract}
We recently identified genes and molecular pathways related to radioresistance of oral squamous cell carcinoma (OSCC) using Affymetrix GeneChip. The current study focused on the association between one of the target genes, intercellular adhesion molecule 2 (ICAM2), and resistance to X-ray irradiation in OSCC cells, and evaluated the antitumor efficacy of combining ICAM2 small interfering RNA (siRNA) and X-ray irradiation. Downregulation of ICAM2 expression by siRNA enhanced radiosensitivity of OSCC cells with the increased apoptotic phenotype via phosphorylation (ser473) of AKT and activation of caspase-3. Moreover, overexpression of ICAM2 induced greater OSCC cell resistance to the $X$-ray irradiation with the radioresistance phenotype. These results suggested that ICAM2 silencing is closely related to sensitivity of OSCC cells to radiotherapy, and that ICAM2 may be an effective radiotherapeutic target for this disease.

British Journal of Cancer (2008) 98, I357-1365. doi:I0.1038/sj.bjc.6604290 www.bjcancer.com

Published online 18 March 2008

(c) 2008 Cancer Research UK
\end{abstract}

Keywords: oral squamous cell carcinoma; radioresistance; ICAM2; siRNA

Radiation therapy has played an important role in controlling tumour growth in many patients with cancer. In patients with oral squamous cell carcinoma (OSCC), radiation therapy is currently the standard adjuvant treatment. However, radiation therapy is sometimes ineffective as cancer cells can be resistant to radiation.

Our previous study showed that the radiosensitivity of OSCC cell lines differs greatly in their response to X-ray radiation, as assessed by clonogenic survival assay (Higo et al, 2005). In this study, we showed that in six OSCC cell lines HSC2 was the most radioresistant and HSC3 was the most radiosensitive cell line. Furthermore, we showed that 25 genes and their related molecular pathways related to cell growth and antiapoptosis were strongly associated with radiation resistance in OSCC (Ishigami et al, 2007). Of them, six were reported to be related to cell proliferation and antiapoptosis, that is, ID1 (Nakajima et al, 1998; Zhang et al, 2004b), ID3 (Plowright et al, 2000; Kowanetz et al, 2004), FGFR3 (Gómez-Román et al, 2005), PEG10 (Okabe et al, 2003; Tsou et al, 2003; $\mathrm{Hu}$ et al, 2004), intercellular adhesion molecule 2 (ICAM2) (Perez et al, 2002), and MMP13 (McCawley and Matrisian, 2000; Chang and Werb, 2001; Corte et al, 2005), and we validated that the expression levels increased substantially in radioresistant cells compared with radiosensitive cells. Among the six genes identified, ICAM2, whose mRNA level in unirradiated HSC2 (radioresistant cells compared with unirradiated HSC3 (radiosensitive) cells was

*Correspondence: Dr K Uzawa; E-mail: uzawak@faculty.chiba-u.jp Revised 3I January 2008; accepted I February 2008; published online I8 March 2008 highest in our previous microarray results (Ishigami et al, 2007), underwent further functional analysis.

Intercellular adhesion molecule 2 has been thought to play a role in lymphocyte recirculation by blocking LFA-1-dependent cell adhesion and mediating adhesive interactions important for an antigen-specific immune response, NK cell-mediated clearance, lymphocyte recirculation, and other cellular interactions important for immune response and surveillance (Staunton et al, 1989; Li et al, 1995; Helander et al, 1996; Carpenito et al, 1997; Lehmann et al, 2003). However, ICAM2 has been reported to be a mediator for a survival signal sufficient to block apoptosis by activation of the PI3K/AKT pathway (Perez et al, 2002). In addition, another recent study has reported that absence of ICAM2 expression resulted in impaired angiogenesis in vitro and in vivo, and that ICAM2-deficient cells were defective in in vitro migration and increased apoptosis (Huang et al, 2005). It has been shown that ICAM2-expressing cells may be resistant to apoptosis induced by anticancer agents, including radiation. Therefore, we hypothesised that ICAM2 inhibitors could enhance the effect of radiation on cancer cells that constitutively express ICAM2. Small-interfering RNA has been used widely to silence gene expression and has been evaluated as an attractive tool for use in therapeutics of many cancers (Gao et al, 2005; Zhang et al, 2005; Amarzguioui et al, 2006; Halder et al, 2006; Hosaka et al, 2006). So, we evaluated whether radiosensitivity of oral cancer cells is determined by cellular ICAM2 by using siRNA targeted against ICAM2 gene. Furthermore, we analysed the radiosensitivity of OSCC cells by upregulating ICAM2 gene expression using expression vector encoding ICAM2 cDNA. 


\section{MATERIALS AND METHODS}

\section{Cell lines and culture conditions}

The human OSCC-derived cell lines HSC2 and HSC3 (Human Science Research Resources Bank, Osaka, Japan) were prepared for this study. The cells were maintained in Dulbecco's modified Eagle's medium (DMEM) (Sigma Chemical Co., St Louis, MO, USA) supplemented with $10 \%$ heat-inactivated foetal bovine serum and $50 \mathrm{U} \mathrm{ml}^{-1}$ penicillin and streptomycin. All cultures were grown at $37^{\circ} \mathrm{C}$ under a humidified atmosphere of $5 \%$ carbon dioxide for routine growth.

\section{siRNA, transfection reagents, and transfection of siRNAs in HSC2 (radioresistant) cells}

Small-interfering RNAs were obtained from Dharmacon Research Inc. (Lafayette, CO, USA). SMART pool siRNA targeting ICAM2 consists of four siRNAs targeting multiple sites on ICAM2 (ICAM2-siRNAs). The sequences for ICAM2-siRNAs are 5'-AAGC AGGAGUCAAUGAAUU-3', 5'-UAACCAGCCUGAAGUGGGU-3', $5^{\prime}$-UGAGAAGGUAUUCGAGGUA- ${ }^{\prime}$, and $5^{\prime}$-ACGAACAGGCUCAG UGGAA-3' (siGENOME SMARTpool, M-11182-00-0005, Human ICAM2, NM_000873). Positive- and negative-control siRNAs were purchased from Dharmacon. Two negative controls were used in this study, that is, vehicle control (treated with only DharmaFECT1 reagent) and siCONTROL non-targeting siRNA pool (D-001210-0105; non-targeting siRNA (siNT)). Cyclophilin $\beta$ (siCONTROL Cyclophilin $\beta$ siRNA, D-001136-01-05) was used as positive silencing control to ascertain transfection efficiency. Cells were transfected with siRNAs using DharmaFECT1 reagent (Dharmacon).

To confirm whether ICAM2 gene is related to radioresistance, we performed an siRNA experiment to inhibit the expression of ICAM2 in HSC2 (radioresistant) cell line that previously reported the expression of ICAM2 as being higher than HSC3 (radiosensitive) cell line (Ishigami et al, 2007). HSC2 Cells were plated in antibiotic-free DMEM at a density of 200000 cells $4 \mathrm{ml}^{-1}$ in $60-\mathrm{mm}$ dishes. After $24 \mathrm{~h}$, the cells were transfected with $100 \mathrm{nmoll}^{-1}$ siRNA in DharmaFECT1 reagent according to the manufacturer's instructions. Briefly, $8 \mu \mathrm{l}$ DharmaFECT1 was diluted in $392 \mu \mathrm{l}$ of serum-free medium and incubated at room temperature for $5 \mathrm{~min}$. In a separate tube, $200 \mu \mathrm{l}$ of $2 \mu \mathrm{moll}^{-1}$ siRNA was diluted in $200 \mu \mathrm{l}$ of serum-free medium at room temperature for $5 \mathrm{~min}$. Diluted DharmaFECT1 $(400 \mu \mathrm{l})$ was added to the diluted siRNA and the complex was incubated for $20 \mathrm{~min}$ at room temperature. The cells were washed with antibiotic-free DMEM and $3.2 \mathrm{ml}$ antibiotic-free DMEM was added to each dish. Small interfering RNA + DharmaFECT1 complex $(800 \mu \mathrm{l})$ was added gently to the dish. The final concentration of siRNA was $100 \mathrm{nmoll}^{-1}$. Control cells were treated with the only medium (HSC2 control), the same amount of transfection reagents (vehicle control), the $100 \mathrm{nmoll}^{-1}$ siNT (negative control), and the $100 \mathrm{nmoll}^{-1}$ cyclophilin $\beta$ siRNA (siCyclophilin $\beta$ ) (positive silencing control). After $4 \mathrm{~h}$ of transfection, the medium of cells treated with ICAM2-siRNAs (siRNA targeted to ICAM2 (siICAM2)) and control cells was replaced with fresh medium, and these were incubated at $37^{\circ} \mathrm{C}$ in $5 \% \mathrm{CO} 2$ for $48 \mathrm{~h}$ to $120 \mathrm{~h}$ before performing experiments.

\section{Transient transfection of ICAM2 DNA}

To verify whether ICAM2 gene is related to radioresistance, we performed overexpression of ICAM2 gene in HSC3 (radiosensitive) cell line that previously reported the expression of ICAM2 as being higher than HSC2 (radioresistant) cell line (Ishigami et al, 2007). Human ICAM2 cDNA was cloned into a pME18SFL3 expression vector (TOYOBO, Osaka, Japan) for transient transfection experiments. HSC3 cell lines were transfected with pME18SFL3 encoding ICAM2 cDNA using the FuGENE HD transfection reagent (Roche
Diagnostics GmbH, Mannheim, Germany). Mock transfection of HSC 3 cell line cultures with the FuGENE HD transfection reagent alone was used as vehicle controls. Transfection efficiency was confirmed by real-time quantitative reverse transcriptase-polymerase chain reaction ( $\mathrm{qRT}-\mathrm{PCR}$ ) and western blot analysis. These analyses were performed as described below.

\section{Irradiation using X-ray}

The cells were irradiated with four single radiation doses $(2,4,6$, and $8 \mathrm{~Gy}$ ) using $\mathrm{X}$-ray irradiation equipment (MBR-1520R-3; Hitachi, Tokyo, Japan) operated at $150 \mathrm{~V}$ and $20 \mathrm{~mA}$ with $\mathrm{AL}$ filtration, at a dose of $2.1 \mathrm{~Gy} \mathrm{~min}^{-1}$.

\section{Isolation of RNA}

Total RNA was extracted from X-ray-irradiated and unirradiated cells with TRIzor reagent (Invitrogen Life Technologies, Carlsbad, CA, USA) according to the manufacturer's instructions. The quality of the total RNA was determined using Bioanalyzer (Agilent Technologies, Palo Alto, CA, USA).

\section{Preparation of cDNA}

Total RNA was extracted from cells using TRIzor reagent. Five micrograms of total RNA of each sample were reversed transcribed to cDNA using Ready-To-Go You-Prime First-Strand Beads (GE Healthcare, Little Chalfort, Buckinghamshire, UK) and oligo (dT) primer (Sigma Genosys, Ishikari, Japan), according to the manufacturers' protocol.

\section{Analysis of mRNA expression by real-time qRT - PCR}

Quantitative reverse transcriptase-polymerase chain reaction was performed to validate mRNA expression with a single method using a LightCycler FastStart DNA Master SYBR Green I kit (Roche Diagnostics $\mathrm{GmbH}$ ), according to the procedure provided by the manufacturer. The oligonucleotides used as primers were $5^{\prime}$-GATC CAGGGCGGAGACTTC- $3^{\prime}$ and $5^{\prime}$-GCCCGTAGTGCTTCAGTTTG A-3' for Cyclophilin $\beta$ mRNA, $5^{\prime}$-CATCTCTGCCCCCTCTGCTGA$3^{\prime}$ and $5^{\prime}$-GGATGACCTTGCCCACAGCCT- $3^{\prime}$ for glyceraldehyde-3phosphate dehydrogenase (GAPDH) mRNA, and $5^{\prime}$-ATTCAACAG CACGGCTGACA- $3^{\prime}$ and $5^{\prime}$-CAGGCTCATAGATCTCCAACATC T- $3^{\prime}$ for ICAM2 mRNA. Using LightCycler apparatus, we carried out PCR reactions in a final volume of $20 \mu \mathrm{l}$ of a reaction mixture consisting of $2 \mu \mathrm{l}$ of FastStart DNA Master SYBR Green I mix, $3 \mathrm{~mm}$ $\mathrm{MgCl}_{2}$, and $1 \mu \mathrm{l}$ of the primers, according to the manufacturer's instructions. Subsequently, the reaction mixture was loaded into glass capillary tubes and subjected to initial denaturation at $95^{\circ} \mathrm{C}$ for $10 \mathrm{~min}$, followed by $33-45$ rounds of amplification at $95^{\circ} \mathrm{C}$ (10 s) for denaturation, $62-68^{\circ} \mathrm{C}(10 \mathrm{~s})$ for annealing, and $72^{\circ} \mathrm{C}$ for extension, with a temperature slope of $20^{\circ} \mathrm{C} \mathrm{s}^{-1}$, performed with LightCycler. The transcript amount for the genes was estimated from the respective standard curves and normalised to the GAPDH transcript amount determined in the corresponding samples.

\section{Protein extraction}

Protein was extracted from the cells, which were washed twice with phosphate-buffered saline, scraped into a tube with lysis buffer ( $7 \mathrm{~m}$ urea, $2 \mathrm{~m}$ thiourea, $4 \% \mathrm{w} / \mathrm{v}$ CHAPS, and $10 \mathrm{~mm}$ Tris, $\mathrm{pH} 8$ ), and incubated at $4{ }^{\circ} \mathrm{C}$ for $10 \mathrm{~min}$. Cell extracts were lysed by sonication $(3 \times 10$-s pulses on ice) and centrifuged at $13000 \mathrm{~g}$ for $10 \mathrm{~min}$ at $4{ }^{\circ} \mathrm{C}$. The supernatant containing the cell proteins then was recovered. Protein concentration was determined using a commercial Bradford reagent (Bio-Rad, Richmond, CA, USA) and adjusted to $1 \mathrm{mg} \mathrm{ml}^{-1}$ with lysis buffer. 


\section{Western blot analysis}

Protein extracts $(15 \mu \mathrm{g})$ were electrophoresed on $12.5 \%$ sodium dodecyl sulfate-polyacrylamide gel electrophoresis gels, transferred to polyvinylidene difluoride (PVDF) membrane (Bio-Rad, Hercules, CA, USA), and blocked for $1 \mathrm{~h}$ at room temperature in $0.3 \%$ skimmed milk. Immunoblot PVDF membranes were washed with Tris-buffered saline Tween-20 (TBST: $10 \mathrm{~mm}$ Tris- $\mathrm{HCl}(\mathrm{pH}$ $8.5), 150 \mathrm{~mm} \mathrm{NaCl}$, and $0.1 \%$ Tween-20) thrice and probed with $2 \mu \mathrm{g} \mathrm{ml}^{-1}$ affinity-purified mouse anti-human ICAM2 monoclonal antibody (R\&D Systems Inc., Minneapolis, MN, USA), $1 \mu \mathrm{g} \mathrm{ml}^{-1}$ affinity-purified rabbit anti-human/mouse/rat specific AKT antibody (Rockland Inc., Gilbertsville, PA, USA), and $1 \mu \mathrm{g} \mathrm{ml}^{-1}$ affinity-purified mouse anti-human p-AKT (pS437) antibody (BIOMOL International, L.P., Plymouth Meeting, PA, USA) overnight at room temperature. For cyclophilin $\beta$ protein and $\beta$-actin protein, $1 \mu \mathrm{g} \mathrm{ml}^{-1}$ affinity-purified rabbit anti-human cyclophilin $\beta$ polyclonal antibody (Abcam Ltd, Cambridge, UK) and $1 \mu \mathrm{g} \mathrm{ml}^{-1}$ affinity-purified mouse anti-human $\beta$-actin monoclonal antibody (Abcam Ltd) were used for $2 \mathrm{~h}$ at room temperature. Polyvinylidene difluoride membranes were washed again and incubated with 1:10000 of Envision + (Dako Japan Inc., Kyoto, Japan) as secondary antibody coupled to horseradish peroxidase-conjugated anti-mouse or ant-rabbit IgG antibody for $2 \mathrm{~h}$ at room temperature. Finally, membranes were incubated using the ECL Plus Western Blotting Detection System (GE Healthcare, Carpinteria, Buckinghamshire, UK) and immunoblotting result was visualised by exposing the membrane to Fuji Medica X-Ray film RX-U (FUJIFILM, Kanagawa, Japan). Signal intensities were quantitated using ImageJ software (1.38x version)(NIH, Bethesda, MD, USA).

\section{Cell-proliferation assay}

To determine the effect of ICAM2 siRNA on cell proliferation, HSC2 cells transfected with non-targeting or ICAM2 siRNA $\left(100 \mathrm{nmoll}^{-1}\right)$ were seeded in 12 -well plates at a density of $1 \times 10^{4}$ viable cells per well. Mock-transfected cells were treated with DharmaFECT1 reagent as vehicle controls but not siRNA. At the indicated time point, cells were trypsinised and counted using a haemocytometer in triplicate samples. The effect on cell proliferation was investigated in the same manner as HSC3 cells treated with expression vector of ICAM2 DNA.

\section{Clonogenic cell survival assay}

HSC2 cells were transfected as above with the vehicle, siNT, and siICAM2. At 72, 84, and $96 \mathrm{~h}$ after transfection, the cells were trypsinised, counted, and the appropriate number of cells were plated in $60-\mathrm{mm}$ dishes and allowed to attach for $24 \mathrm{~h}$. After $24 \mathrm{~h}$, the cells were irradiated $(2,4,6,8 \mathrm{~Gy})$ and incubated for 8-10 days. The colonies were stained with crystal violet (Sigma Chemical Co.), and colonies of 50 cells or greater were counted. Clonogenic fractions of irradiated cells were normalised to the plating efficiency of unirradiated controls. Likewise, colonies of HSC3 cells transfected with expression vector of ICAM2 DNA were measured.

\section{In situ cell apoptosis detection assay}

HSC2 cells were plated in 24-well dishes at a density of $2 \times 10^{4}$ cells per well. After $24 \mathrm{~h}$, HSC2 cells were treated with siRNA (vehicle, siNT, siICAM2). After incubation for $120 \mathrm{~h}$, cells were analysed for apoptosis by terminal deoxynucleotidyl transferase (TdT)mediated dUTP nick-end labelling (TUNEL) procedure, using an in situ apoptosis detection kit (Takara Bio, Shiga, Japan). Briefly, cells were fixed in $4 \%$ paraformaldehyde for $15 \mathrm{~min}$. After treatment with permeabilisation buffer (Takara Bio) for 2 min on ice, the cells were incubated with TdT enzymes plus labeling safe buffer (Takara Bio) at $37^{\circ} \mathrm{C}$. After $1.5 \mathrm{~h}$, the cells were washed with phosphate-buffered saline and observed by fluorescence microscopy. Cells undergoing apoptosis were specifically labelled with fluorescein-dUTP with high sensitivity, allowing immediate detection by fluorescence microscopy. Apoptotic cells were counted and expressed as a percentage of the total cells. The experiments were performed in triplicate.

\section{Caspase- 3 activity assay}

We performed three different assays for caspase-3 activity. Two assays (using caspase-3 detection kit; Calbiochem, Oxford, UK) measured the amount of activated caspase- 3 in situ in living cells by a caspase- 3 inhibitor (DEVD-FMK) conjugated to FITC as a fluorescent in situ marker. Fluorescein isothiocyanate-DEVD-FMK is cell-permeable and nontoxic and irreversibly binds to activated caspase- 3 in apoptotic cells. The FITC label allows direct detection of activated caspase- 3 by fluorescence microscopy or a fluorescence microplate reader. In our initial experiment, FITC fluorescence was conjugated to the inhibitor, thereby registering a fluorescent label detected by fluorescence microscopy in cells in which caspase- 3 had been activated. Both cells treated with and without ICAM2 siRNA (control, vehicle, and siNT) were compared. Caspase- 3 active cells were counted and expressed as a percentage of the total number of cells. The experiments were performed in triplicate. For the next experiment, we used a microplate reader to study the effect. Data were expressed as the difference in activity among the four data points (control, vehicle, siNT, and siICAM2) and indicated the amount of caspase- 3 activity that remained. The kit was used according to the manufacturer's instructions. Briefly, cells were treated with or without ICAM2 siRNA for $120 \mathrm{~h}$, after which they (about $1 \times 10^{6} \mathrm{ml}^{-1}$ ) were incubated for $1 \mathrm{~h}$ with FITCDEVD-FMK at $37^{\circ} \mathrm{C}$. The cells then were washed twice by washing buffer and then the fluorescence intensity was measured by fluorescence microscopy and using a fluorescence microplate reader (Wallac 1420 ARVOsx Multilabel Counter; Perkin-Elmer, Chiba, Japan), with excitation at approximately $485 \mathrm{~nm}$ and emission detection at approximately $535 \mathrm{~nm}$. Finally, we performed activated caspase- 3 western blot analysis. Monoclonal antibody to active caspase-3 (Genlantis Inc., San Diego, CA, USA) was used for detection. Western blot analysis for activated caspase3 was performed as described previously.

\section{RESULTS}

\section{Transfection efficiency of siRNAs}

To ascertain whether conditions for RNA inhibition were optimal and that transfection efficiency was satisfactory, siCyclophilin $\beta$ was used as a positive silencing control. In real-time qRT-PCR analysis, cyclophilin $\beta$ mRNA expression decreased by $69 \%$ with siCyclophilin $\beta 48 \mathrm{~h}$ after transfection (Figure 1A). In western blot analysis, cyclophilin $\beta$ was detected as a single band. In cells transfected with siCyclophilin $\beta$, the band diminished significantly $(P<0.01$, Student's $t$-test $)$, confirming high transfection efficiency $120 \mathrm{~h}$ after transfection compared with the vehicle, siCONTROL siNT, and the non-transfected control cells. HSC2 cells transfected with siCyclophilin $\beta$ showed little change in the band of cyclophilin $\beta 96$ and $108 \mathrm{~h}$ after transfection compared with controls (Figure 1B).

\section{ICAM2 protein levels in HSC2 cells transfected with siICAM2 and control siRNAs}

Figure 1C shows western blot analysis of ICAM2 protein expression in HSC2 cells $120 \mathrm{~h}$ after transfection with siRNAs. This time point was selected by a positive silencing control test in an siRNA transfection experiment (Figure 1B). The ICAM2 protein levels in cells transfected with vehicle, siNT, and siICAM2- 

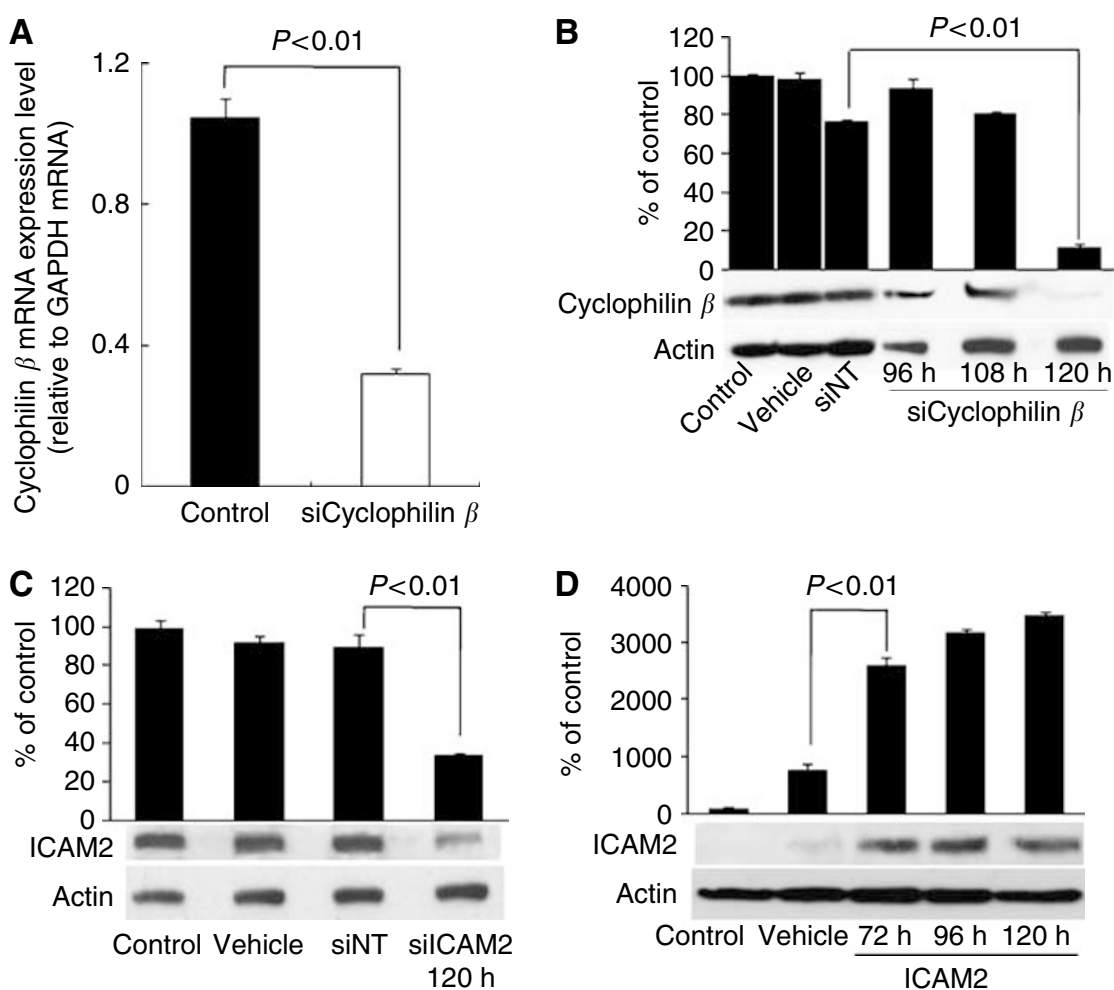

Figure I Effect of siRNAs. (A) Expression of cyclophilin $\beta$ mRNA in non-transfected HSC2 cells (control) and HSC2 cells transfected with siCyclophilin $\beta$ $\left(P<0.0\right.$ I, Student's t-test). (B) Western blot analysis of cyclophilin $\beta$ protein (positive control) in HSC2. The cells were transfected with I00 nmol I ${ }^{-1}$ siNT and siCyclophilin $\beta$ and analysed at 96, 108, and 120 h. Cyclophilin $\beta$ and actin bands were scanned and quantitated as described under Materials and Methods. The values obtained from densitometric analysis of each cyclophilin $\beta$ protein first were normalised to actin protein levels and then expressed as the percentage of the values of control. Cyclophilin $\beta$ proteins were significantly inhibited $(P<0.0$ I, Student's $t$-test) in cells transfected with siCyclophilin $\beta$ at I $20 \mathrm{~h}$. There is no change in cyclophilin $\beta$ in cells transfected with vehicle and siNT negative controls siRNA. (C) Western blot analysis of ICAM2 protein in HSC2 cells transfected with vehicle, siNT, and silCAM2. The cells were transfected with $100 \mathrm{nmol}^{-1}$ siRNAs and analysed at $120 \mathrm{~h}$. Intercellular adhesion molecule 2 and actin bands were scanned and quantitated as described under Materials and Methods. The values obtained from densitometric analysis of each ICAM2 protein first were normalised to actin protein levels and then expressed as the percentage of the values of control. The ICAM2 proteins were significantly inhibited $(P<0.01$, Student's t-test) in cells transfected with silCAM2 at I20 h. (D) Western blot analysis of ICAM2 in ICAM2-overexpressing HSC3 cells. The cells were examined 72, 96, and $120 \mathrm{~h}$ after transient transfection of expression vector encoding ICAM2 cDNA. Actin was used as a loading control. Intercellular adhesion molecule 2 and actin bands were scanned and quantitated as described under Materials and Methods. The values obtained from densitometric analysis of each ICAM2 protein first were normalised to actin protein levels and then expressed as the percentage of the values of control. The ICAM2 protein levels increased significantly $(P<0.0$ I, Student's $t$-test) in cells transfected with the expression vector of ICAM2 DNA at 72 , 96 and $120 \mathrm{~h}$

transfected cells were comparable to those in non-transfected cells. In cells transfected with $100 \mathrm{nmoll}^{-1}$ silCAM2, ICAM2 protein level decreased significantly $(P<0.01$, Student's $t$-test) compared with non-transfected, vehicle-transfected, and the siNT-transfected cells (Figure 1C). In addition, qRT-PCR analysis showed that ICAM 2 mRNA expression decreased by 67,97 , and $96 \%$ in HSC2 cells with siICAM2 compared with non-transfected cells at 24, 48, and $72 \mathrm{~h}$, respectively, after transfection.

\section{ICAM2 protein levels in HSC3 cells transfected with ICAM2 DNA}

Figure 1D shows western blot analysis of ICAM2 protein expression in HSC3 cells 72,96 , and $120 \mathrm{~h}$ after transfection with expression vector encoding ICAM2 cDNA. The ICAM2 protein level increased significantly $(P<0.01$, Student's $t$-test $)$ in cells transfected with ICAM2 DNA compared with vehicle and nontransfected control cells (Figure 1D). Furthermore, qRT-PCR analysis showed that the level of ICAM2 mRNA expression increased significantly $(P<0.01$, Student's $t$-test $)$ by $4.5 \times 10^{6}$ and $9.4 \times 10^{6}$-fold in HSC3 cells with expression vector encoding ICAM2 cDNA compared with non-transfected cells 48 and $72 \mathrm{~h}$, respectively, after transfection.

\section{Effect of ICAM2 siRNA and expression vector encoding ICAM2 cDNA on cell growth}

To determine the effect of ICAM2 siRNA and expression vector encoding ICAM2 cDNA on the growth of the cancer cell lines, a series of cell growth experiments was carried out. The growth curves of HSC2 cells showed that treatment with ICAM2 siRNA inhibited cell growth over a period of 6 days, but cell growth was not inhibited by vehicle and siNT (Figure 2A). Furthermore, the growth curve of HSC3 cells showed that treatment with expression vector encoding ICAM 2 cDNA increased cell growth over a period of 6 days compared with the vehicle (Figure 2B).

\section{Radiosensitivity of HSC2 cells transfected with siICAM2}

The plating efficiencies of unirradiated HSC2 cells (mean \pm s.d., $n=3)$ were $0.278 \pm 0.038$ (control), $0.270 \pm 0.032$ (vehicle), $0.262 \pm 0.031$ (siNT), $0.258 \pm 0.031$ (silCAM2, $120 \mathrm{~h}), 0.275 \pm 0.066$ $($ siICAM2, $108 \mathrm{~h})$, and $0.220 \pm 0.057$ (siICAM2, 96h). The difference in the plating efficiency of siICAM2 cells compared with vehicle or siNT cells was not significant. Figure $2 \mathrm{C}$ shows the radiation survival curves of untreated control cells and cells transfected with siRNAs. These data were used to calculate dose $\mathrm{D}_{37}$ that is required to reduce survival to $37 \%$ (Toulany et al, 2006). 

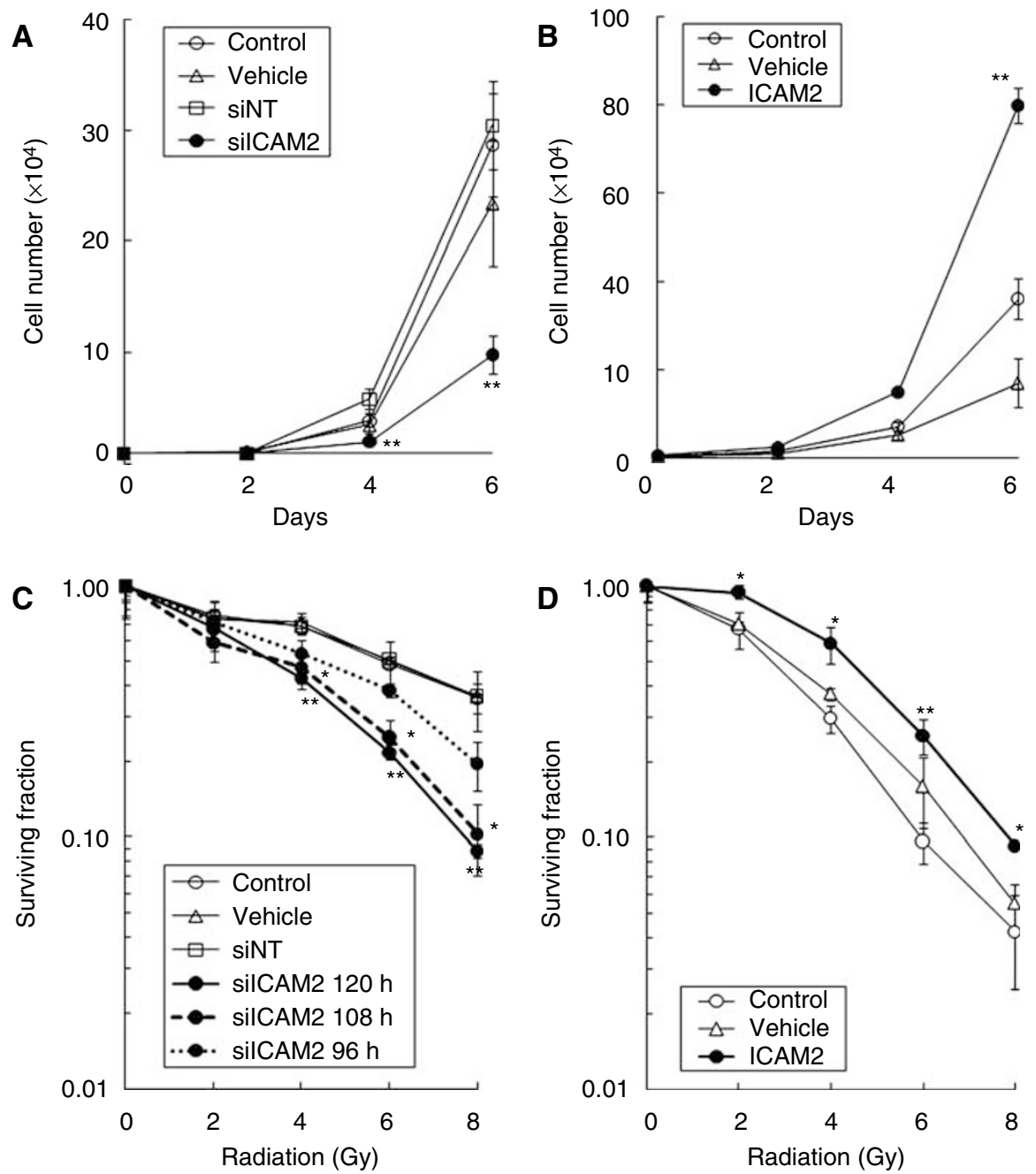

Figure 2 Effect of ICAM2 expression on cell growth and cell survival. (A) HSC2 cells were transfected with I00 nmol I'- ICAM2 siRNA (silCAM2), siNT, or vehicle. At the indicated time points, the cells were trypsinised and counted using a haemocytometer in triplicate samples. The results represent the mean \pm s.d. The asterisks indicate significant differences between siNT and ICAM2 siRNA (**P $<0.0$ I, Student's t-test). (B) HSC3 cells were transfected with expression vector encoding ICAM2 CDNA or vehicle. At the indicated time points, the cells were trypsinised and counted using a haemocytometer in triplicate samples. The results are expressed as the mean \pm s.d. The asterisks indicate significant differences between the vehicle and ICAM2 transfectant (** $P<0.0$ I, Student's t-test). (C) The effect of radiation on the clonogenic survival of HSC2 cells transfected with the vehicle, siNT, and silCAM2. At 72, 84, and $96 \mathrm{~h}$ after transfection; the cells were trypsinised and plated for clonogenic survival assay for silCAM2 at $96 \mathrm{~h}$, silCAM2 at $108 \mathrm{~h}$, and silCAM2 at I20 h, respectively. After $24 \mathrm{~h}$, when the cells had attached, they were irradiated. Colonies were stained with crystal violet and counted after $8-10$ days. Each experiment was repeated at least three times; the error bars represent \pm s.d. The significance of the difference between siNT (open square) and silCAM2 (closed circle) is indicated by asterisks (**P $<0.01$, $* P<0.05$, Student's $t$-test). (D) The effect of radiation on the clonogenic survival of HSC3 cells overexpressing ICAM2, vehicle treated cells, and untreated control. The clonogenic survival of HSC3 cells was calculated in the same way as for HSC2 cells. The significance of the difference between the vehicle (open triangles) and ICAM2-overexpressing cells (closed circles) is indicated by asterisks (*** $<0.01$, $* P<0.05$, Student's t-test).

For HSC2 cells, dose $\mathrm{D}_{37}$ values for control, vehicle, siNT, siICAM2 at $108 \mathrm{~h}$, siICAM2 at $96 \mathrm{~h}$, and siICAM2 at $120 \mathrm{~h}$ were 7.6, 7.6, 7.7, $6.0,4.7$, and $4.4 \mathrm{~Gy}$, respectively. Compared with the vehicletreated control, at the $37 \%$ survival level, the radiosensitivity of cells transfected with siICAM2 at $120 \mathrm{~h}$ was enhanced by a dosemodifying factor of 1.73 and the radiosensitivity of cells transfected with siNT was 0.99 -fold.

\section{Cells transfected with siICAM2 at $120 \mathrm{~h}$ compared with cells transfected with siNT}

The radiosensitivity of cells transfected with siICAM2 at $120 \mathrm{~h}$ was enhanced by a dose-modifying factor of 1.75 for siNT. Moreover, survival of HSC2 cells transfected with siICAM2 at $120 \mathrm{~h}$ decreased significantly $(P<0.01$, Student's $t$-test $)$ after 4,6 , and 8 Gy of radiation compared with that of HSC2 cells treated with siNT (Figure 2C). This indicates that radiation-induced sublethal damage is severe when ICAM2 expression is inhibited.

\section{Radiosensitivity of HSC3 cells transfected with expression vector of ICAM2 DNA}

In HSC3 cells, the plating efficiencies at $0 \mathrm{~Gy}$ (mean \pm s.d., $n=3$ ) were $0.211 \pm 0.030$ (control), $0.262 \pm 0.003$ (vehicle), and $0.232 \pm 0.030$ (ICAM2). The difference in the plating efficiency of cells transfected with expression vector of ICAM2 DNA, compared with vehicle or untreated control cells, was not significant. The radiation survival curves of cells are shown in Figure 2D. In cells transfected with expression vector encoding ICAM2 cDNA, compared with cells transfected with vehicle and untreated control 
cells, the radiosensitivity of the transfectant decreased. For HSC3 cells, dose $\mathrm{D}_{37}$ values for control, vehicle, and the transfectant were $3.4,4$, and $5 \mathrm{~Gy}$, respectively. The resulting dose-modifying factors were 0.80 for the vehicle and 0.68 for the untreated control cells at the $37 \%$ survival level. Furthermore, the rate of cell survival of the transfectant increased significantly $\left({ }^{* *} P<0.01,{ }^{\star} P<0.05\right.$, Student's $t$-test) after 2, 4, 6, and $8 \mathrm{~Gy}$ of radiation, compared with that of cells treated with vehicle (Figure 2D).

\section{Correlation of ICAM2 expression and apoptosis}

To examine whether expression of ICAM2 is the result of apoptosis, we analysed DNA fragmentation by TUNEL assay. As shown in Figure $3 \mathrm{~A}-\mathrm{D}$, positive TUNEL labelling in apoptotic cells was bright. Figure 3D clearly shows that apoptosis was induced in some cells but not in others (Figure $3 \mathrm{~A}-\mathrm{C}$ ). The percentages of apoptotic cells in HSC2 cells transfected with the vehicle, siNT,
siICAM2, and in the untreated control cells were $1.40 \pm 0.09$, $2.56 \pm 0.29,26.4 \pm 8.81$, and $1.05 \pm 0.14 \%$, respectively. Data are expressed as the mean \pm s.d. of three independent experiments. The difference between siNT and silCAM2 reached significance $(P<0.01$, Student's $t$-test $)$

\section{ICAM2-induced AKT phosphorylation}

Recent studies have reported that ICAM2 activated the protein kinase B (PKB)/AKT pathway leading to inhibition of apoptosis (Perez et al, 2002). In this pathway, phosphorylation (Ser473) of AKT protein induced antiapoptosis. To investigate the correlation of ICAM2 and AKT phosphorylation, we performed western blot analysis with antibody recognising AKT and AKT phosphorylation at Ser473 site (Figure 3I). Figure 3I shows that HSC2 cells treated with silCAM2 resulted in lower AKT phosphorylation level than cells treated with the vehicle, siNT, and the untreated control cells.
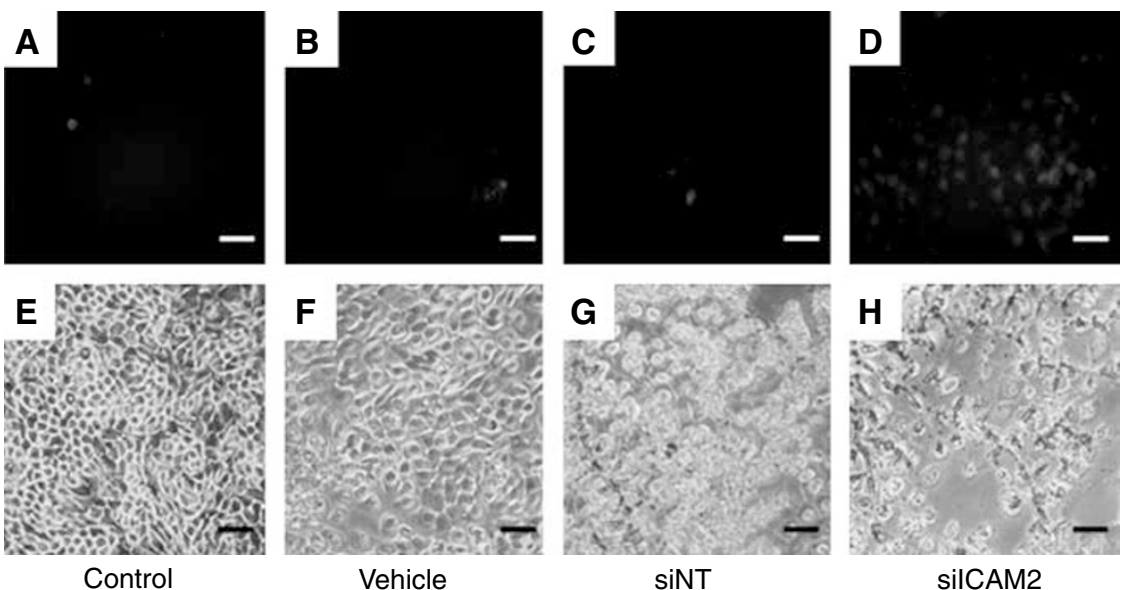

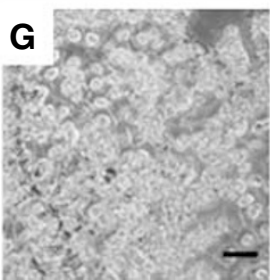

siNT

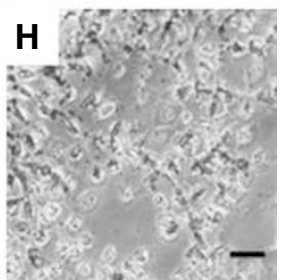

silCAM2

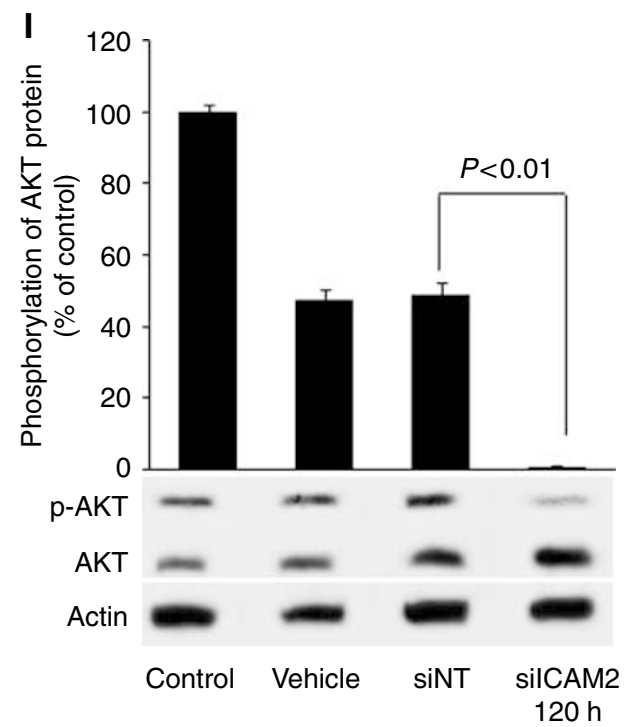

Figure 3 The effects of ICAM2 inhibition on apoptosis in HSC2 cells. DNA fragmentation of apoptotic cells is detected by TUNEL assay. (D) HSC2 cells were treated with $100 \mathrm{nmol}^{-1}$ silCAM2 for $120 \mathrm{~h}$. Many apoptotic cells are detected in HSC2 cells with silCAM2. (A-C) Few apoptotic cells are detected in cells transfected with the vehicle, siNT, and in untreated control cells. $(\mathbf{E}-\mathbf{H})$ The lower panels are phase-contrast micrographs showing total cells, Bar $=35 \mu \mathrm{m}$. (I) Western blot analyses of AKT and p-AKT (AKT phosphorylation at the Ser473 site) protein in HSC2 cells transfected with the vehicle, siNT, silCAM2, and in untreated control. The cells were transfected with $100 \mathrm{nmol}^{-1}$ siRNAs and analysed at I $20 \mathrm{~h}$. p-AKT and AKT bands were scanned and quantitated as described under Materials and Methods. The values obtained from densitometric analysis for each AKT proteins were first normalised to AKT protein levels and then expressed as the percentage of the values for the control. The $P$-AKT proteins were significantly inhibited $(P<0.01$, Student's $t$-test) in cells transfected with silCAM2 at $120 \mathrm{~h}$ 

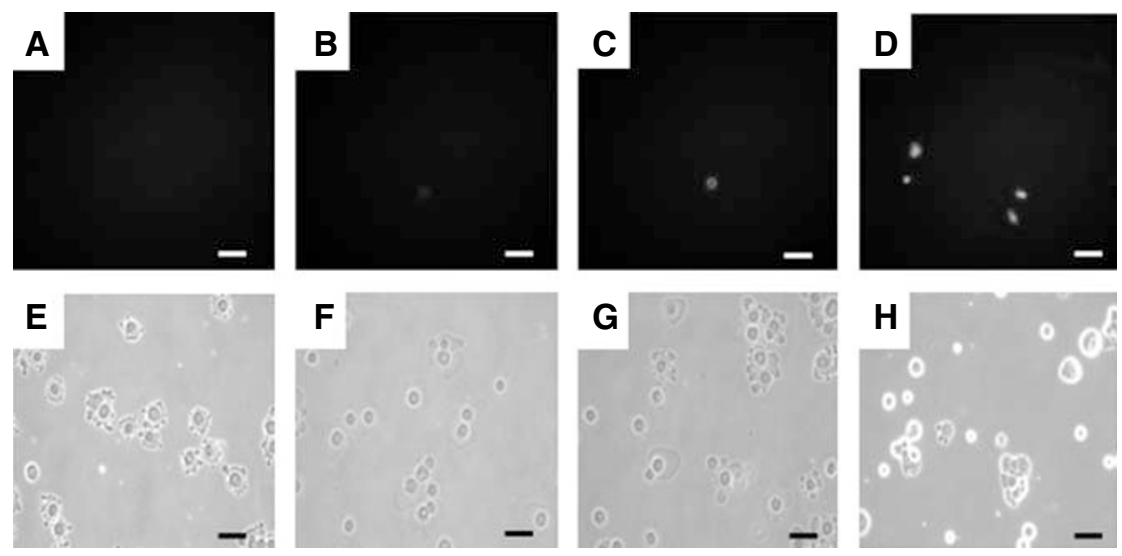

Control

Vehicle

siNT

SilCAM2
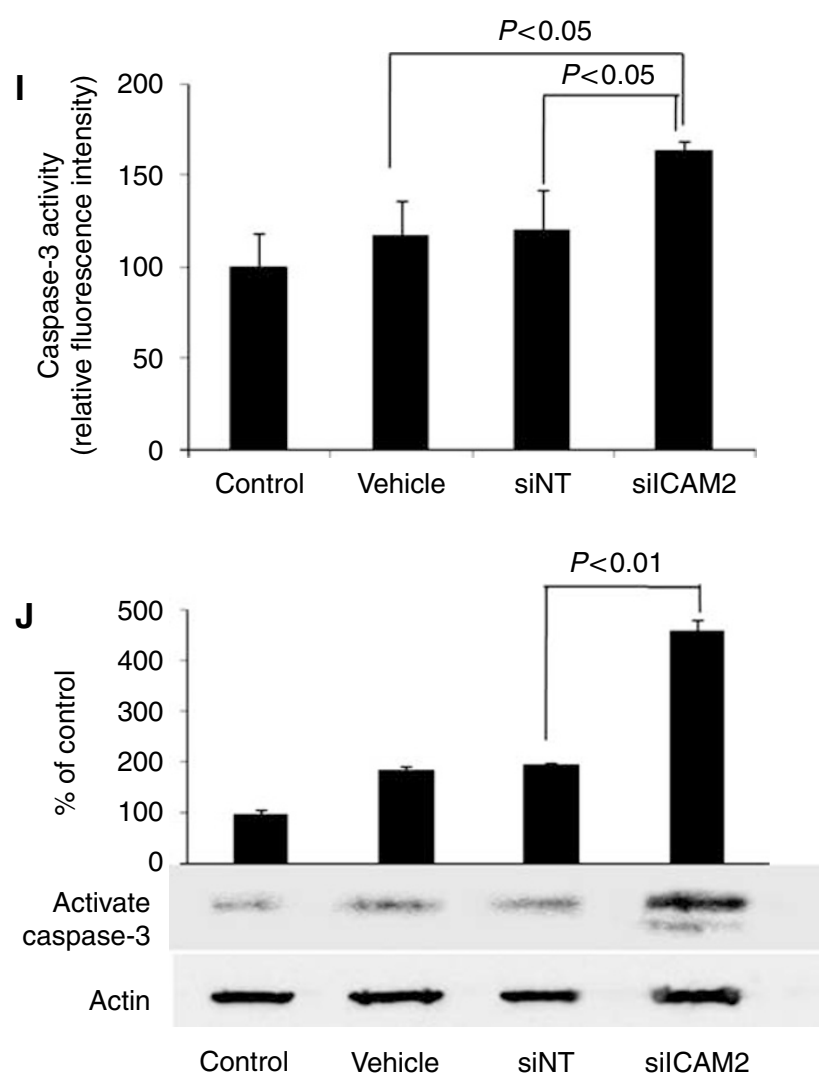

Figure 4 The effect of ICAM2 inhibition on caspase-3 activity in HSC2 cells. (D) HSC2 cells treated with $100 \mathrm{nmol} \mathrm{I}^{-1}$ silCAM2 for I $20 \mathrm{~h}$. Relatively more caspase-3 active cells are detected in HSC2 cells with silCAM2. (A-C) Few caspase-3 active cells are detected in cells transfected with the vehicle, siNT, and in untreated control cells. $(\mathbf{E}-\mathbf{H})$ The lower panels are phase-contrast micrographs showing total cells. Bar $=35 \mu \mathrm{m}$. (I) HSC2 cells were treated with ICAM2 siRNA for $120 \mathrm{~h}$ and the activity of caspase- 3 was measured using the caspase-3 detection kit with a microplate reader as described under Materials and Methods. The results are expressed as mean \pm s.d. of three independent experiments. Caspase-3 activity is increased significantly $(P<0.05$, Student's $t-$ test) in cells transfected with silCAM2 at $120 \mathrm{~h}$. (J) Western blot analysis of active caspase-3 protein in HSC2 cells transfected with the vehicle, siNT, and silCAM2. Cells were transfected with $100 \mathrm{nmol} \mathrm{I}^{-1}$ siRNAs and analysed at $120 \mathrm{~h}$. Active caspase-3 and actin bands were scanned and quantitated as described under Materials and Methods. The values obtained from densitometric analysis for each active caspase-3 protein were first normalised to actin protein levels and expressed as the percentage of the control values. Active caspase-3 protein levels are increased significantly $(P<0.01$, Student's $t$-test) in cells transfected with silCAM2 at $120 \mathrm{~h}$.

\section{Caspase- 3 activity assay}

We investigated the role of caspase- 3 in this process to determine the mechanism by which apoptosis occurred. Figure $4 \mathrm{~A}-\mathrm{D}$ show the green FITC signal present within the siICAM2 cells; control, vehicle, and siNT cells displayed a small activated caspase- 3 signal. The percentages of caspase- 3 active cells in HSC2 cells transfected with the vehicle, siNT, siICAM2, and the untreated control cells were $2.60 \pm 2.30 \%, 4.00 \pm 0.61 \%, 20.7 \pm 0.71 \%$, and $1.45 \pm 2.51 \%$, respectively. Data are expressed as the mean \pm s.d. of three independent experiments. The difference between siNT and silCAM2 reached significance $(P<0.01$, Student's $t$-test $)$. The second assay (caspase-3 activity assay) (Figure $4 \mathrm{I}$ ) showed that caspase- 3 activity was significantly elevated in cells with ICAM2 siRNA for $120 \mathrm{~h}$ compared with cells with vehicle and siNT $(P<0.05$, Student's $t$-test $)$. Quantification of relative fluorescence 
intensity revealed that siICAM2 increased caspase-3 enzymatic activity 1.36 -fold compared with siNT. The third study examined the protein levels of active caspase-3. Figure $4 \mathrm{~J}$ shows that HSC2 cells treated with siICAM2 had significantly higher $(P<0.01$, Student's $t$-test) active caspase- 3 protein levels than cells treated with the vehicle, siNT, and the untreated control cells.

\section{DISCUSSION}

ICAM2, whose mRNA level was upregulated in radioresistant OSCC cells in our previous study (Ishigami et al, 2007), was one of candidate genes selected as radioresistant genes of OSCC cells by microarray analysis using Affymetrix GeneChip.

The current study was designed to examine whether ICAM2 is functionally associated with radiosensitivity of OSCC in vitro. In the previous report, little has been mentioned about the role of ICAMs in regard to radioresistance. Intercellular adhesion molecule 1 expression was shown to be elevated by hypoxia and radiation (Zünd et al, 1997; Meineke et al, 2002). Intercellular adhesion molecule 3 expression was reported to associate with radioresistance in cervical cancer (Chung et al, 2005).

In our study, the results indicated that ICAM2 inhibition induces radiosensitive in vitro (Figure 2C). A further important point is that radiosensitisation of cells may depend on the extent of ICAM2 protein inhibition. Because HSC2 cells were incubated for a longer time with siICAM2, they became more radiosensitive (Figure 2C), suggesting that radiosensitisation of HSC2 cells may be determined by the amount of ICAM2 protein present in the cells at the time of irradiation. Moreover, ICAM2 overexpression induces radioresistance in vitro (Figure 2D). These results may indicate that regulation of ICAM2 protein is correlated with radiosensitivity. Further, Figure $2 \mathrm{~A}$ and $\mathrm{B}$ show that regulation of ICAM2 protein is related to cell growth. The question that needs to be answered is what mechanisms play a role in the relation between the regulation of the ICAM2 protein and these results.

Intercellular adhesion molecule 2-induced activation of AKT kinase resulted in the activation of several downstream effectors as detected by phosphorylation of BAD, GSK3, FKHR, and AFX, all of which can contribute to cell survival (Zha et al, 1996; Brunet et al, 1999). The PI3/AKT-signalling system is a general mediator of extracellular stimuli, including growth factors, cytokines, and adhesion, to extracellular matrices (Downward, 1998). Phosphatidylinositol-3,4,5-triphosphate binds to the pleckstrin domain of AKT and recruits AKT to the membrane, where it becomes dually phosphorylated (Alessi et al, 1996) and is then activated and phosphorylates a number of downstream effectors that contribute to cell survival. These studies indicated that ICAM2 activation might lead to an antiapoptic signal in a variety of cells. A recent study identified a pathway of ICAM2 activating the PI3K/AKT leading to inhibition of apoptosis (Perez et al, 2002). This pathway showed that ICAM2 induced tyrosine phosphorylation of ezrin and PI3K kinase membrane translocation, resulting in phosphatidyli- nositol-3,4,5 production, PDK-1 and AKT activation, and subsequent phosphorylation of AKT targets BAD, GSK3, and FKHR. The previous studies may have presumed that the differences in cell growth and radiosensitivity are caused by mechanisms of antiapoptotic effect induced by ICAM2. Consequently, we postulated that ICAM2 induced antiapoptosis in OSCCs as well as the mechanism of the $\mathrm{PKB} / \mathrm{AKT}$ pathway activation previously reported (Perez et al, 2002). In addition, AKT phosphorylation at Ser473 was suggested to be correlated perfectly with antiapoptosis in lymphocytes (Perez et al, 2002). Therefore, we examined AKT phosphorylation (Ser473) and cell apoptosis to confirm the relation between ICAM2 and antiapoptosis via the PKB/AKT pathway in OSCC cells. The results of our experiment showed that cells with silCAM2 induced a decrease in AKT phosphorylation (Ser473) (Figure 3I). To detect cell apoptosis, we performed caspase- 3 activity assays and TUNEL assay. We evaluated caspase3 activation in cells treated with ICAM2 siRNA. HSC2 cells treated with silCAM2 showed increased caspase- 3 activity compared with untreated or control cells (Figure 4). In the previous studies, the apoptotic pathway induced by decrease in p-AKT caused activation of caspase-3 (Ivins Zito et al, 2004; Zhang et al, 2004a; Zhuang et al, 2007). We considered that decreased p-AKT and increased activated caspase- 3 induced apoptosis in cells treated with ICAM2 siRNA. Furthermore, results of the TUNEL assay showed increased apoptosis (Figure 3D). Accordingly, it is reasonable that inhibiting ICAM2 may contribute to radiosensitisation of OSCC cells by increased apoptosis via phosphorylation (Ser473) of AKT and activation of caspase-3.

Our study was similar to a previous study (Chung et al, 2005) in which the investigators used the same methods, that is, microarray analysis, siRNA, overexpression, and apoptosis assay. These two studies yielded similar results in that they both confirmed a relation between a gene and radioresistance. However, due to availability of clinical samples, our study differed from the other in that we could not analyse clinical specimens from patients. Our study, therefore, may be insufficient in vivo. Our study could not prove a relation between ICAM2 and radioresistance in OSCC cells in vitro.

In conclusion, ICAM2 expression may be associated with radioresistance in OSCC cells, ICAM2 siRNA may enhance the radiosensitivity of oral cancer cells, and ICAM2 may be an effective radiotherapeutic target of oral cancer and a marker for radiation sensitivity based on in vitro studies with microarray analysis.

\section{ACKNOWLEDGEMENTS}

We thank Lynda C Charters for editing this paper. This work was supported by a Grant-in-Aid for Japan Society for Promotion of Science Fellows (19-52163), a Grant-in-Aid for Exploratory Research (19659520), and The 21st Century Center of Excellence (COE) Programs grant.

\section{REFERENCES}

Alessi D, Andjelkovic M, Caudwell B, Cron P, Morrice N, Cohen P, Hemmings B (1996) Mechanism of activation of protein kinase B by insulin and IGF-1. EMBO J 15: 6541-6551

Amarzguioui M, Peng Q, Wiiger M, Vasovic V, Babaie E, Holen T, Nesland J, Prydz $\mathrm{H}$ (2006) Ex vivo and in vivo delivery of anti-tissue factor short interfering RNA inhibits mouse pulmonary metastasis of B16 melanoma cells. Clin Cancer Res 12: 4055-4061

Brunet A, Bonni A, Zigmond $\mathrm{M}$, Lin $\mathrm{M}$, Juo $\mathrm{P}, \mathrm{Hu} \mathrm{L}$, Anderson $\mathrm{M}$, Arden K, Blenis J, Greenberg M (1999) Akt promotes cell survival by phosphorylating and inhibiting a Forkhead transcription factor. Cell 96: $857-868$
Carpenito C, Pyszniak A, Takei F (1997) ICAM-2 provides a costimulatory signal for T cell stimulation by allogeneic class II MHC. Scand J Immunol 45: $248-254$

Chang C, Werb Z (2001) The many faces of metalloproteases: cell growth, invasion, angiogenesis and metastasis. Trends Cell Biol 11: S37-S43

Chung Y, Kim B, Park C, Huh S, Kim J, Park J, Cho S, Kim B, Kim J, Yoo Y, Bae D (2005) Increased expression of ICAM-3 is associated with radiation resistance in cervical cancer. Int J Cancer 117: 194-201

Corte M, Gonzalez L, Corte M, Quintela I, Pidal I, Bongera M, Vizoso F (2005) Collagenase-3 (MMP-13) expression in cutaneous malignant melanoma. Int J Biol Markers 20: 242-248 
Downward J (1998) Mechanisms and consequences of activation of protein kinase B/Akt. Curr Opin Cell Biol 10: 262-267

Gao L, Zhang L, Hu J, Li F, Shao Y, Zhao D, Kalvakolanu D, Kopecko D, Zhao X, Xu D (2005) Downregulation of signal transducer and activator of transcription 3 expression using vector-based small interfering RNAs suppresses growth of human prostate tumor in vivo. Clin Cancer Res 11: $6333-6341$

Gómez-Román J, Saenz P, Molina M, Cuevas González J, Escuredo K, Santa Cruz S, Junquera C, Simón L, Martínez A, Gutiérrez Baños J, López-Brea M, Esparza C, Val-Bernal J (2005) Fibroblast growth factor receptor 3 is overexpressed in urinary tract carcinomas and modulates the neoplastic cell growth. Clin Cancer Res 11: 459-465

Halder J, Kamat A, Landen CJ, Han L, Lutgendorf S, Lin Y, Merritt W, Jennings N, Chavez-Reyes A, Coleman R, Gershenson D, Schmandt R, Cole S, Lopez-Berestein G, Sood A (2006) Focal adhesion kinase targeting using in vivo short interfering RNA delivery in neutral liposomes for ovarian carcinoma therapy. Clin Cancer Res 12: 4916-4924

Helander T, Carpén O, Turunen O, Kovanen P, Vaheri A, Timonen T (1996) ICAM-2 redistributed by ezrin as a target for killer cells. Nature 382: $265-268$

Higo M, Uzawa K, Kouzu Y, Bukawa H, Nimura Y, Seki N, Tanzawa H (2005) Identification of candidate radioresistant genes in human squamous cell carcinoma cells through gene expression analysis using DNA microarrays. Oncol Rep 14: 1293-1298

Hosaka S, Nakatsura T, Tsukamoto H, Hatayama T, Baba H, Nishimura Y (2006) Synthetic small interfering RNA targeting heat shock protein 105 induces apoptosis of various cancer cells both in vitro and in vivo. Cancer Sci 97: 623-632

Hu C, Xiong J, Zhang L, Huang B, Zhang Q, Li Q, Yang M, Wu Y, Wu Q, Shen Q, Gao Q, Zhang K, Sun Z, Liu J, Jin Y, Tan J (2004) PEG10 activation by co-stimulation of CXCR5 and CCR7 essentially contributes to resistance to apoptosis in CD19+CD34+ B cells from patients with $\mathrm{B}$ cell lineage acute and chronic lymphocytic leukemia. Cell Mol Immunol 1: $280-294$

Huang M, Mason J, Birdsey G, Amsellem V, Gerwin N, Haskard D, Ridley A, Randi A (2005) Endothelial intercellular adhesion molecule (ICAM)-2 regulates angiogenesis. Blood 106: $1636-1643$

Ishigami T, Uzawa K, Higo M, Nomura H, Saito K, Kato Y, Nakashima D, Shiiba M, Bukawa H, Yokoe H, Kawata T, Ito H, Tanzawa H (2007) Genes and molecular pathways related to radioresistance of oral squamous cell carcinoma cells. Int J Cancer 120: 2262-2270

Ivins Zito C, Kontaridis M, Fornaro M, Feng G, Bennett A (2004) SHP-2 regulates the phosphatidylinositide $3^{\prime}$-kinase/Akt pathway and suppresses caspase 3-mediated apoptosis. J Cell Physiol 199: 227-236

Kowanetz M, Valcourt U, Bergström R, Heldin C, Moustakas A (2004) Id2 and Id3 define the potency of cell proliferation and differentiation responses to transforming growth factor beta and bone morphogenetic protein. Mol Cell Biol 24: 4241-4254

Lehmann J, Jablonski-Westrich D, Haubold U, Gutierrez-Ramos J, Springer T, Hamann A (2003) Overlapping and selective roles of endothelial intercellular adhesion molecule-1 (ICAM-1) and ICAM-2 in lymphocyte trafficking. J Immunol 171: 2588-2593

Li R, Xie J, Kantor C, Koistinen V, Altieri D, Nortamo P, Gahmberg C (1995) A peptide derived from the intercellular adhesion molecule-2 regulates the avidity of the leukocyte integrins $\mathrm{CD} 11 \mathrm{~b} / \mathrm{CD} 18$ and $\mathrm{CD} 11 \mathrm{c} /$ CD18. J Cell Biol 129: 1143-1153

McCawley L, Matrisian L (2000) Matrix metalloproteinases: multifunctional contributors to tumor progression. Mol Med Today 6: 149-156

Meineke V, Moede T, Gilbertz K, Mayerhofer A, Ring J, Köhn F, Van Beuningen D (2002) Protein kinase inhibitors modulate time-dependent effects of UV and ionizing irradiation on ICAM-1 expression on human hepatoma cells. Int J Radiat Biol 78: 577-583

Nakajima T, Yageta M, Shiotsu K, Morita K, Suzuki M, Tomooka Y, Oda K (1998) Suppression of adenovirus E1A-induced apoptosis by mutated p53 is overcome by coexpression with Id proteins. Proc Natl Acad Sci USA 95: 10590 - 10595

Okabe H, Satoh S, Furukawa Y, Kato T, Hasegawa S, Nakajima Y, Yamaoka Y, Nakamura Y (2003) Involvement of PEG10 in human hepatocellular carcinogenesis through interaction with SIAH1. Cancer Res 63: $3043-3048$

Perez O, Kinoshita S, Hitoshi Y, Payan D, Kitamura T, Nolan G, Lorens J (2002) Activation of the PKB/AKT pathway by ICAM-2. Immunity 16: $51-65$

Plowright E, Li Z, Bergsagel P, Chesi M, Barber D, Branch D, Hawley R, Stewart A (2000) Ectopic expression of fibroblast growth factor receptor 3 promotes myeloma cell proliferation and prevents apoptosis. Blood 95: $992-998$

Staunton D, Dustin M, Springer T (1989) Functional cloning of ICAM-2, a cell adhesion ligand for LFA-1 homologous to ICAM-1. Nature 339: 61 - 64

Toulany M, Kasten-Pisula U, Brammer I, Wang S, Chen J, Dittmann K, Baumann M, Dikomey E, Rodemann H (2006) Blockage of epidermal growth factor receptor-phosphatidylinositol 3-kinase-AKT signaling increases radiosensitivity of K-RAS mutated human tumor cells in vitro by affecting DNA repair. Clin Cancer Res 12: 4119-4126

Tsou A, Chuang Y, Su J, Yang C, Liao Y, Liu W, Chiu J, Chou C (2003) Overexpression of a novel imprinted gene, PEG10, in human hepatocellular carcinoma and in regenerating mouse livers. J Biomed Sci 10: $625-635$

Zha J, Harada H, Yang E, Jockel J, Korsmeyer S (1996) Serine phosphorylation of death agonist BAD in response to survival factor results in binding to 14-3-3 not BCL-X(L). Cell 87: 619-628

Zhang H, Rao J, Guo X, Liu L, Zou T, Turner D, Wang J (2004a) Akt kinase activation blocks apoptosis in intestinal epithelial cells by inhibiting caspase-3 after polyamine depletion. J Biol Chem 279: 22539-22547

Zhang X, Chen Z, Choe M, Lin Y, Sun S, Wieand H, Shin H, Chen A, Khuri F, Shin D (2005) Tumor growth inhibition by simultaneously blocking epidermal growth factor receptor and cyclooxygenase-2 in a xenograft model. Clin Cancer Res 11: 6261-6269

Zhang X, Ling M, Feng H, Wong Y, Tsao S, Wang X (2004b) Id-I stimulates cell proliferation through activation of EGFR in ovarian cancer cells. $\mathrm{Br} \mathrm{J}$ Cancer 91: $2042-2047$

Zhuang S, Yan Y, Daubert R, Han J, Schnellmann R (2007) ERK promotes hydrogen peroxide-induced apoptosis through caspase-3 activation and inhibition of Akt in renal epithelial cells. Am J Physiol Renal Physiol 292: F440 - F447

Zünd G, Uezono S, Stahl G, Dzus A, McGowan F, Hickey P, Colgan S (1997) Hypoxia enhances induction of endothelial ICAM-1: role for metabolic acidosis and proteasomes. Am J Physiol 273: C1571-C1580 